\section{Convergent evolution: limited forms most beautiful}

\author{
David A. Liberles \\ Department of Molecular Biology, \\ University of Wyoming, USA
}

\section{Book Review}

Convergent Evolution: Limited Forms Most Beautiful by George McGhee attempts the first systematic examination of the timely topic of convergent evolution. The author's expertise and passion really lie in studies of morphology and behavior and these trajectories are the strongest of the work. Starting (Chapter 1) with an introduction to convergent evolution rooted in current thought in the systematics community, the author moves through a cata$\log$ of animals (Chapter 2) and plants (Chapter 3 ) that show evidence for some form of convergence. While the compilation of examples is impressive, some of the examples presented seem somewhat trivial, like the evolution of fast running, feeding on insects, or the generation of commercially produced fruit that is popular with human consumers. These examples run counter to the general theme that there is a strong role for constraint in limiting the evolvability of species. On this last point, it has controversially been suggested that domesticatability is limited and that few species are actually domesticatable, ${ }^{1}$ suggesting much more limited scope for changes in organismal phenotype and niche than is widely appreciated. Scientifically this view is still at the stage of a hypothesis that is not fully tested or accepted.

The following chapter (Chapter 4) describes Convergent Ecosystems, but is not really about ecosystems so much as organisms filling niches. A periodic table of niches is presented and along these lines describes organismal life history attributes rather than niches from a truly ecological perspective. Chapter 5 describes Convergent Molecules, while Chapter 6 describes Convergent Minds, a chapter on animal behavior rather than neurobiology. These chapters are all treated independently, whereas a multi-scale mechanistic framework would have been more powerful in describing the potential for convergence. Morphology has its roots in molecular biology, which in turn has its roots in the biophysical chemistry of macromolecular folding and interaction. Behavior also has its links running from genes to neuronal structures to minds and ultimately behavior. The mappings across layers of organization can be informative to what is really possible and what is really convergent.
Running fast can be achieved in many different ways, and even the most diehard traditional systematist might not expect running speed to be a good phylogenetic character. Further, even for something more concrete like coat pigmentation, is this convergent evolution if different genes have undergone substitution to enable the phenotype to evolve? If there are multiple molecular solutions to evolving a given phenotype, should that phenotype really be considered convergent or constraining? Should one insist on multi-level of organization evidence for convergence to truly show that constraint has channeled the biological system to one solution?

However, there is a need for serious thinking about convergent evolution. Systematics is rooted in thought in the search for synapomorphies, conserved clade-defining characteristics, identified using neutral evolutionary models (or parsimony) applied to various molecular and morphological data sets. That such things as invariant synapomorphies exist needs to be questioned and that is the potential benefit of a book like this. However, the existence of invariant synapomorphies is not explicitly questioned in this book. Viewing clades of organisms through observed invariant synapomorphies from sequences sampled is to miss the process of evolution. Evolution is the description of probabilistic change where the probabilities are context dependent. Understanding where these probabilities come from and how they link across layers of biological organization is a major research goal. That there is constraint that affects the probabilities of certain types of change from a given starting point does not mean that the possible solutions are a small set. It has been suggested that biologically sampled sequence space in the observed protein universe is expanding and is not a small set. ${ }^{2}$ To anyone working in protein design, this is perhaps not a surprising result. This extends to non-observed morphological states, like the author's example of a 6 legged vertebrate. Just because it hasn't been observed doesn't mean that it is impossible for one reason or another. How do we know that it just hasn't been mutationally accessible at a point when it might not be selected against? Mutational accessibility relates to the chance of hitting upon an evolutionary stable solution from a given starting point and a set of population genetic parameters together with a given fitness landscape dictating selection.

Along these lines, the author is famous for his work in the area of theoretical morphology, the question of what morphological states are possible or impossible. This is a useful intellectual construct, but needs to be placed in the context of the neutral theory, something never mentioned in the book, as well as through the lens of mutational accessibility. Many descriptions are presented as selectionist accounts,
Correspondence: David A. Liberles, Department of Molecular Biology, University of Wyoming, Laramie, WY 82071, USA.

E-mail: liberles@uwyo.edu

Key words: convergent evolution, book review, McGhee.

Received for publication: 22 February 2012. Accepted for publication: 22 February 2012.

This work is licensed under a Creative Commons Attribution NonCommercial 3.0 License (CC BYNC 3.0).

(C) Copyright D.A. Liberles, 2012

Licensee PAGEPress srl, Italy

Trends in Evolutionary Biology 2012; 4:e2 doi:10.4081/eb.2012.e2

with no description of the role of parameters like effective population size ( $\mathrm{Ne})^{3-4}$ in modulating the power of selection or in the idea of constructive neutralism..$^{5-6}$ Mutations occur at the level of DNA, which is why a multi-layer accounting is desirable in generating a mechanistic understanding of convergent evolution. When are convergent solutions necessitated by selection and constraint and when are they hit upon by chance, given underlying mutational probabilities?

Further, it is perhaps curious that the author does not discuss bacteria or protozoans. It is unclear how theoretical morphology would be applied to this, but there is a diverse biochemistry in bacteria, some of which is likely convergent. Going from molecular convergence of enzymes to the roles of different bacterial and protozoan lineages in similar roles in various ecosystems would enable a multilayer synthesis that is much harder to present from the perspective of animals and plants. While the competition of birds and bats is conceptually interesting, there is now metagenomic data that might allow testing of hypotheses of competition and niche convergence between various micro-organisms. Further, the existence of horizontal gene transfer in microbial lineages as a very different mechanism from mutation in the context of vertical descent emphasizes the importance of linking layers of biological organization in the study of convergent evolution.

I am perhaps giving Professor McGhee short shrift. The strongest chapters in the book, Chapters 7 and 8, do present a discussion of the intersection of developmental and functional constraint from a morphological perspective. While the author may be over-estimating the strength and role of selection in enabling and eliminating various morphological forms to create a limited viable space, it is clear that convergent evolution does happen 
and is something that needs to be taken more seriously by those in the systematics and evolutionary biology communities.

As a last point, Chapters 2, 3 and 5 lacked figures. If a second edition of the book is ever produced, pictures of various convergent animals, plants, and proteins would complement the extensive lists compiled by the author. As the first book on the topic, it is a valuable introduction to convergent evolution that research will expand upon by examining links between bio-macromolecules, brain structures, morphology, behavior, and ecosystems to understand the role of selection and constraint in evolution.

\section{References}

1. Diamond J. Evolution, consequences and future of plant and animal domestication. Nature 2002;418:700-7.

2. Povolotskaya IS, Kondrashov FA. Sequence space and the ongoing expansion of the protein universe. Nature 2010;465:922-6.
3. Lynch M. The frailty of adaptive hypotheses for the origins of organismal complexity. Proc Natl Acad Sci USA 2007;104 Suppl1:8597-604.

4. Lynch M. The Origins of Genome Architecture. Sunderland, MA: Sinauer Associates, Inc; 2007.

5. Stoltzfus A. On the possibility of constructive neutral evolution. J Mol Evol 1999;49: 169-81.

6. Gray MW, Lukes J, Archibald JM, et al. Cell biology. Irremediable complexity? Science 2010;330:920-1. 